\title{
Analytical Solutions of a Class of Optimum Orbit Modifications ${ }^{1}$
}

\author{
N. X. VINH ${ }^{2}$ AND C. MARChaL ${ }^{3}$ \\ Communicated by J. V. Breakwell
}

\begin{abstract}
This paper presents the complete analytical solution of several fundamental problems in orbital correction. The initial orbit is represented by a given point in the phase space, while the final orbit is constrained to stay in a given curve which can be bounded, unbounded, or composed of a finite number of segments of different curves. The inclusion of atmospheric maneuver as part of the optimum process is discussed; its analy tical treatment can be carried out by modifying the final state to include the set of orbits having their perigee at the boundary of the atmosphere.

The selection of the apogee and perigee distances as state variables gives a symmetric form to the problem and results in a linear differential equation of the first order for the ratio of the adjoint variables. The introduction of a curve of comparison, called the separatrix, facilitates the discussion of the existence of a corner on an optimal trajectory.
\end{abstract}

\section{Introduction}

Consider a space vehicle initially in an orbit $E_{0}$ around a spherical planet with center of attraction at $O$. The initial orbit is defined by its semimajor axis $a_{0}$ and its eccentricity $e_{0}$. It is proposed to bring the vehicle, by a series of orbital maneuvers, into a final orbit such that its elements, denoted by the subscript 1 , satisfy a relation of the form

$$
f\left(a_{1}, e_{1}\right)=0
$$

${ }^{1}$ Paper received August 22, 1969. This work was supported by NASA Contract No. NASr 54(06).

${ }^{2}$ Associate Professor, Department of Aerospace Engineering, The University of Michigan, Ann Arbor, Michigan.

${ }^{s}$ Staff Scientist, ONERA, Chatillon, France. 


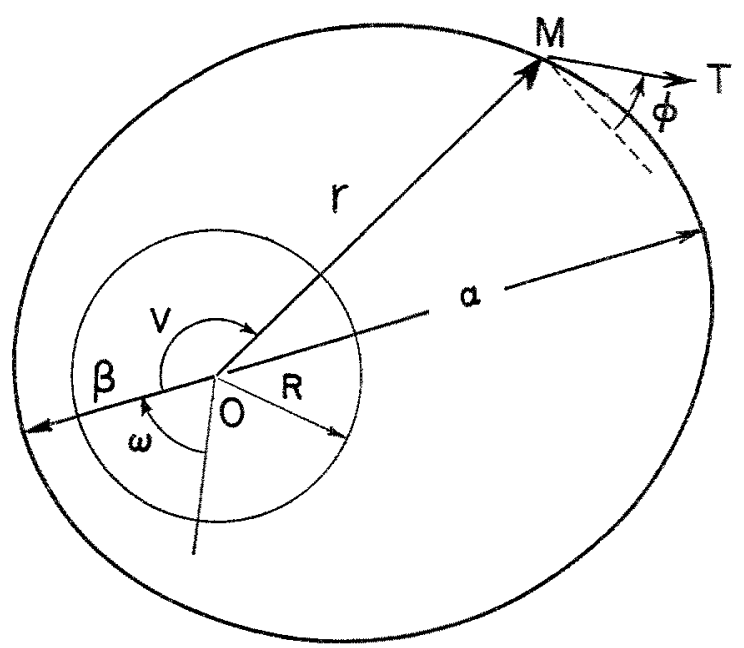

Fig. 1. Osculating orbit.

We seek to minimize the total characteristic velocity for the maneuver. Since, for a high-thrust propulsion system, the characteristic velocity provides a direct measure of the fuel consumption, the optimal trajectory considered in this paper yields the minimum fuel expenditure.

We assume that the planet is surrounded by a spherical atmosphere with center at $O$ and radius $R$ (Fig. 1). In the search of the absolute minimum fuel consumption, we further assume that the duration of the maneuver is unlimited and that the thrust provided by the rockets on board the space vehicle is not bounded, that is, it can produce impulsive changes in the velocity. For the case where the thrust magnitude is limited, it can be made impulsive by the process of fractioning. Thus, the problem is of the class of time-free, impulsive orbital transfers.

\section{Formulation of the Problem}

The problem is formulated as an optimal control problem. At the time $t$, the state of the vehicle is characterized by the row vector (Fig. 1)

$$
\boldsymbol{\xi}=(\alpha, \beta, \omega, u)
$$

where $\alpha$ is the apogee distance, $\beta$ the perigee distance, $\omega$ the longitude of the perigee, and $u$ the characteristic velocity. The first three coordinates describe 
the osculating ellipse along which the vehicle is moving at the time $t$, that is, the Keplerian orbit which the vehicle would follow should the engine cease to operate at the time $t$. The parameter $u$ is a measure of the latent velocity expended since the initial time and is defined by

$$
u=\int_{0}^{t}(T / m) d t \geqslant 0
$$

where $T$ is the instantaneous magnitude of the thrust and $m$ the mass of the vehicle. The control is represented by the row vector

$$
\eta=(v, \phi)
$$

where $v$ is the true anomaly and $\phi$ the thrust direction with respect to the local horizon.

The equations of motion are derived from the classical equations of variations in celestial mechanics (Ref. 1). For a time-free problem, $u$ is a convenient independent variable. We have

$$
\begin{aligned}
\frac{d \alpha}{d u} & =\frac{2 \alpha^{2}}{n b(\alpha+\beta)}\left[\sin v \sin \phi+\frac{(\alpha-\beta) \cos ^{2} v+2(\alpha+\beta) \cos v+(\alpha+3 \beta)}{(\alpha+\beta)+(\alpha-\beta) \cos v} \cos \phi\right] \\
& =f_{1}(\xi, \eta) \\
\frac{d \beta}{d u} & =-\frac{2 \beta^{2}}{n b(\alpha+\beta)}\left[\sin v \sin \phi+\frac{(\alpha-\beta) \cos ^{2} v+2(\alpha+\beta) \cos v-(3 \alpha+\beta)}{(\alpha+\beta)+(\alpha-\beta) \cos v} \cos \phi\right] \\
& =f_{2}(\xi, \eta) \\
\frac{d \omega}{d u} & =-\frac{4 \alpha \beta}{n b\left(\alpha^{2}-\beta^{2}\right)}\left[\cos v \sin \phi-\frac{2(\alpha+\beta)+(\alpha-\beta) \cos v}{(\alpha+\beta)+(\alpha-\beta) \cos v} \sin v \cos \phi\right]=f_{3}(\xi, \eta) \\
\frac{d u}{d u} & =1=f_{0}(\xi, \eta)
\end{aligned}
$$

where

$$
b=\sqrt{ }(\alpha \beta), \quad n=\sqrt{ }\left[8 \mu /(\alpha+\beta)^{3}\right]
$$

respectively denote the semiminor axis and the mean motion, and where $\mu=G M$ is the gravitational constant. The end conditions are

$$
\begin{array}{rlll}
u_{0}=0, & a=\alpha_{0}=a_{0}\left(1+e_{0}\right), & \beta=\beta_{0}=a_{0}\left(1-e_{0}\right), \quad \omega_{0}=0 \\
u & =u_{1}, \quad \alpha=\alpha_{1}, & \beta=\beta_{1}, \quad \omega=\omega_{1} &
\end{array}
$$


and $\alpha_{1}, \beta_{1}$ are such that they satisfy a specified relation

$$
\theta\left(\alpha_{1}, \beta_{1}\right)=0
$$

The problem is to find, at each instant $u$, the control $\eta$ such that the characteristic velocity $u_{1}$ is a minimum. Using the maximum principle, we define an adjoint vector $\lambda=\left(\lambda_{1}, \lambda_{2}, \lambda_{3}\right)$ such that its components satisfy the adjoint equations (Ref. 2)

$$
d \lambda_{1} / d u=-\partial H / \partial \alpha, \quad d \lambda_{2} / d u=-\partial H / \partial \beta, \quad d \lambda_{3} / d u=-\partial H / \partial \omega=0
$$

where the Hamiltonian $H$ is given by

$$
H=\lambda_{0}+\lambda_{1} f_{1}+\lambda_{2} f_{2}+\lambda_{3} f_{3}
$$

with

$$
\lambda_{0}=-1<0
$$

The optimal trajectory is obtained by integrating the system of equations (5) and (9) subject to the end conditions (7), (8), (11), with the control parameters $v$ and $\phi$ selected such that, at each instant, $H$ is an absolute maximum.

\section{Analysis}

3.1. Optimal Trajectories. We note that $\omega$ is an ignorable coordinate. Hence, if the final orientation of the orbit is not specified, $\lambda_{3}=0$; the condition of optimality is the maximization of the reduced Hamiltonian

$$
\bar{H}=\lambda_{1} f_{1}+\lambda_{2} f_{2}
$$

with respect to $v$ and $\phi$.

If the angles $v$ and $\phi$ are not constrained (this situation is considered in this paper), then it is easy to verify that the stationary values of $\bar{H}$ correspond to $v=0$ or $v=\pi$ and $\phi=0$ or $\phi=\pi$. Therefore, along an extremal arc,

$$
\sin v=\sin \phi=0, \quad \cos v= \pm 1=\epsilon_{1}, \quad \cos \phi= \pm 1=\epsilon_{2}
$$

Along an extremal, the Hamiltonian (10) reduces to

$$
H=\lambda_{0}+\frac{4 \epsilon_{2}\left[\left(1+\epsilon_{1}\right) \alpha^{2} \lambda_{1}+\left(1-\epsilon_{1}\right) \beta^{2} \lambda_{2}\right]}{n b\left[(\alpha+\beta)+\epsilon_{1}(\alpha-\beta)\right]}
$$



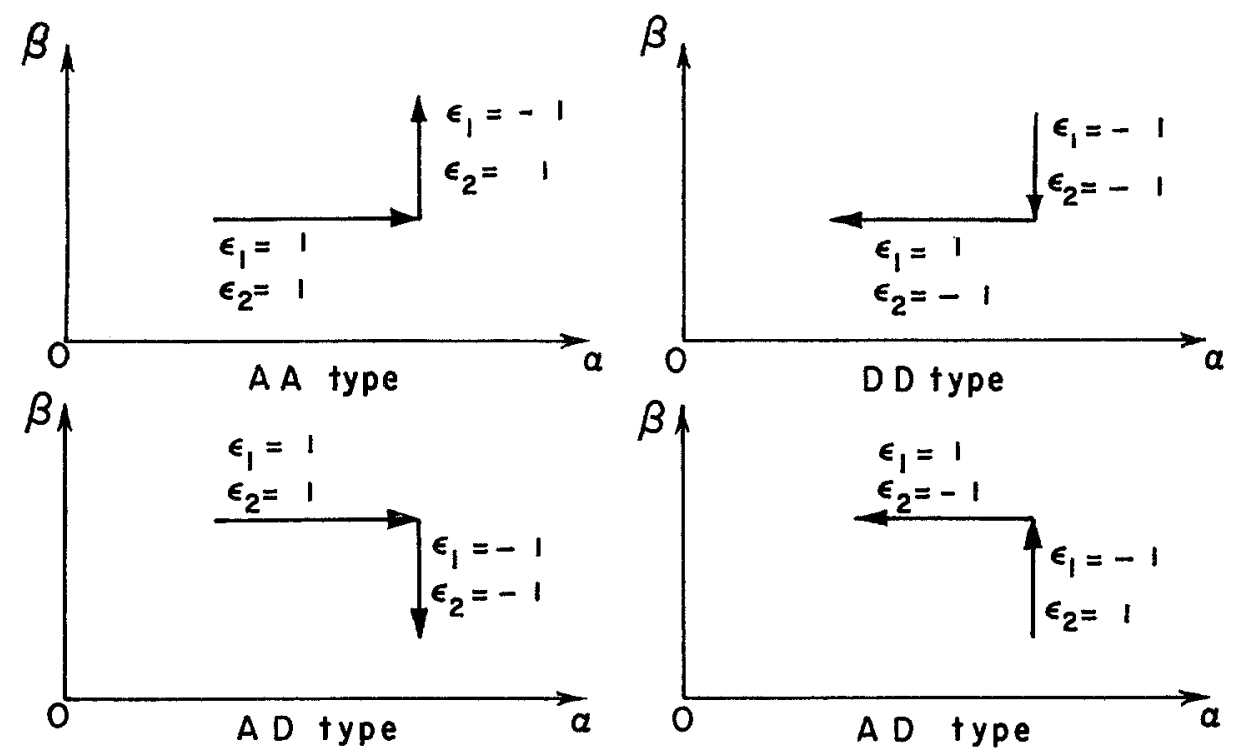

Fig. 2. Different types of switching.

and, by elimination of $u$ in (5), the equation of the optimal trajectory can be written as

$$
\left[\left(1+\epsilon_{1}\right) / \beta^{2}\right] d \beta=\left[\left(1-\epsilon_{1}\right) / \alpha^{2}\right] d \alpha
$$

and implies that

$$
\begin{aligned}
& \alpha=\text { Const } \quad \text { when } \quad \epsilon_{1}=-1 \\
& \beta=\text { Const } \quad \text { when } \quad \epsilon_{1}=1
\end{aligned}
$$

In the $\alpha \beta$-space, with $\alpha \geqslant \beta$, the optimal trajectories are the lines parallel to the axes (Fig. 2). The impulses are always applied tangentially at the apses.

3.2. Switching Curve. Along an optimal trajectory, there may exist a corner $S$ (or switching) at which the trajectory changes direction. The direction of switching is of four possible types, as shown in Fig. 2. Using the letter $A$ to designate an accelerative impulse and $D$ for a decelerative impulse, we have the following types of switching:

First type: $A A$-switching starting from the perigee. On the left of the corner $S, \epsilon_{1}=1, \epsilon_{2}=1$. On the right of the corner, $\epsilon_{1}=-1, \epsilon_{2}=1$. Let

$$
\Psi=\lambda_{1} / \lambda_{2}
$$


By writing that the Hamiltonian (13) is continuous across a corner, we have, for the value of $\Psi$ at the point $S$,

$$
\Psi_{S}=\beta / \alpha
$$

where $\alpha$ and $\beta$ are the coordinates of the point $S$.

Second type: $D D$-switching starting from the apogee. This is the inverse operation of the previous one. On the left of the corner $S$, we have $\epsilon_{1}=1$, $\epsilon_{2}=-1$. On the right, we have $\epsilon_{1}=-1, \epsilon_{2}=-1$. In this case, the value of $\Psi$ at the point $S$ is also given by (16).

Third type: AD-switching starting from the perigee. On the left of the corner $S, \epsilon_{1}=1, \epsilon_{2}=1$. On the right, we have $\epsilon_{1}=-1, \epsilon_{2}=-1$. The value of the ratio $\Psi$ at the point $S$ for this type of switching is

$$
\Psi_{S}=-\beta / \alpha
$$

Fourth type: $A D$-switching starting from the apogee. This is the inverse operation of the preceding one. On the left, we have $\epsilon_{1}=1, \epsilon_{2}=-1$. On the right, we have $\epsilon_{1}=-1, \epsilon_{2}=1$. The value of $\Psi$ at the point $S$ is also given by (17).

For a prescribed final state represented by Eq. (8), the locus of the possible switching point $S$ is a curve, called the switching curve. The switching curve is obtained by integrating the adjoint equations (9) along the last subarc $S K$ (Fig. 3) and using the corner condition at point $S$ and the transversality condition at point $K$. In the figure, the final state is designated by $\Sigma$.

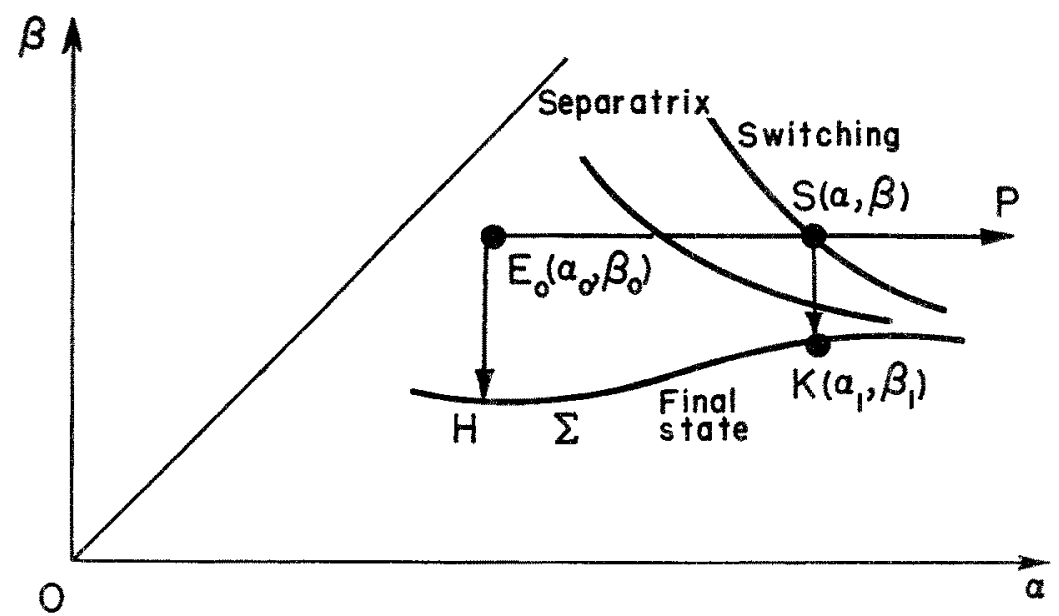

Fig. 3. Switching curve and separatrix. 
Integration along $\alpha=$ Const, $\epsilon_{1}=-1$. Explicitly, we have

$$
\begin{aligned}
d \lambda_{1} / d u & =-\left(2 \epsilon_{2} \lambda_{2} / n b\right)[\beta(2 \alpha-\beta) / \alpha(\alpha+\beta)] \\
d \lambda_{2} / d u & =-\left(2 \epsilon_{2} \lambda_{2} / n b\right)[(4 \beta+\alpha) /(\alpha+\beta)] \\
d \beta / d u & =\left(4 \epsilon_{2} \beta / n b\right)
\end{aligned}
$$

Using $\beta$ as the new independent variable, we have the equation for $\Psi=\lambda_{1} / \lambda_{2}$

$$
d \Psi / d \beta-[(4 \beta+\alpha) / 2 \beta(\alpha+\beta)] \Psi=(\beta-2 \alpha) / 2 \alpha(\alpha+\beta)
$$

The general solution of this equation is

$$
\Psi=C \sqrt{ }\left[\beta(\alpha+\beta)^{3}\right]-\beta(2 \alpha+\beta) / \alpha^{2}
$$

where the constant of integration $C$ is to be determined by the appropriate end conditions.

Since the last subarc is along $\alpha=$ Const, the switching is of the first type or the third type. For an $A A$-type, using the value (16) for $\Psi$, we have for the constant $C$ evaluated at the point $S$

$$
C=\beta(3 \alpha+\beta) / \alpha^{2} \sqrt{ }\left[\beta(\alpha+\beta)^{3}\right]
$$

where $\alpha$ and $\beta$ are the coordinates of the point $S$. For an $A D$-type of switching, the value (17) for $\Psi$ at the point $S$ is used to calculate the constant $C$. We have

$$
C=\beta / \alpha^{2} \sqrt{ }[\beta(\alpha+\beta)]
$$

At the terminal point $K$, the vector $\left(\lambda_{1}, \lambda_{2}\right)$ is orthogonal to the curve $\theta\left(\alpha_{1}, \beta_{1}\right)=0$, by the transversality condition. Then, the value of $\Psi$ at the point $K\left(\alpha_{1}, \beta_{1}\right)$ is

$$
\Psi_{k}=\left(\delta \theta / \delta \alpha_{1}\right) /\left(\delta \theta / \delta \beta_{1}\right)
$$

Using this value to calculate $C$ in (19), we have

$$
C=C\left(\alpha_{1}, \beta_{1}\right)=C\left(\alpha, \beta_{1}\right)
$$

In the last relation, $\beta_{1}$ can be calculated in terms of $\alpha$ by solving

$$
\theta\left(\alpha, \beta_{1}\right)=0
$$

Finally, if the value of $C$ in (23) is equated to the value of $C$ in (20) or (21), depending on the type of switching, we obtain a relation between $\alpha$ and $\beta$, which is the equation of the switching curve. 
Integration along $\beta=$ Const, $\epsilon_{1}=1$. If the last subarc $S K$ is along a line $\beta=$ Const, the adjoint equations are integrated along this line, using $\alpha$ as independent variable. We obtain

$$
1 / \Psi=C \sqrt{ }\left[\alpha(\alpha+\beta)^{3}\right]-\alpha(\alpha+2 \beta) / \beta^{2}
$$

Because of the symmetry of the state variables, this last relation can be easily obtained by replacing $\Psi$ by $1 / \Psi$ in Eq. (19) and interchanging $\alpha$ and $\beta$.

The switching now is of the second type or the fourth type. For a $D D-$ switching, the value of the constant $C$ evaluated at the point $S$ is

$$
C=\alpha(\alpha+3 \beta) / \beta^{2} \sqrt{ }\left[\alpha(\alpha+\beta)^{3}\right]
$$

For an $A D$-switching, we have

$$
C=\alpha / \beta^{2} \sqrt{ }[\alpha(\alpha+\beta)]
$$

At the terminal point $K$, by using the transversality condition (22), we have for the constant $C$ evaluated at $K\left(\alpha_{1}, \beta_{1}\right)$

$$
C=C\left(\alpha_{1}, \beta_{1}\right)=C\left(\alpha_{1}, \beta\right)
$$

In the last relation, $\alpha_{1}$ can be evaluated in terms of $\beta$ by solving

$$
\theta\left(\alpha_{1}, \beta\right)=0
$$

Finally, if the value of $C$ in (28) is equated to the value of $C$ in (26) or (27), depending on the type of switching, we obtain the equation of the switching curve.

In the following, the equation of the switching curve is represented by

$$
S(\alpha, \beta)=0
$$

In deriving the equation of the switching curve, we have assumed that no constraint has been put on the final state. If the final state is constrained, then an optimal trajectory may have a corner which is not on the switching curve. In this case, the final orbit is always at the boundary of the final state. Another type of corner on an optimal trajectory may arise when atmospheric drag is used in the optimal transfer. This type of corner is discussed in Section 3.4.

3.3. Separatrix. The application of the maximum principle only gives the necessary conditions for optimality. Therefore, for a specified problem, even in the case where the switching curve is real in the space $\alpha \geqslant \beta>0$, it 
only means that, if the final state is not constrained and if a corner exists on an optimal trajectory, this corner has to be on the switching curve. To avoid the difficult task of proving the sufficiency for optimality, which requires the finding of the conjugate point, we introduce a curve called the separatrix which can be used to rule out the existence of the corner in most cases. The separatrix is defined as a curve which delimits the domain where a transfer via parabolic orbits is more economical than going directly to the final state by applying an impulse at one of the apses. Like the switching curve, the separatrix depends on the final state. The discussion is illustrated in Fig. 3. For the initial orbit $E_{0}$, the optimal trajectory to reach the final state $\Sigma$ is the trajectory $E_{0} H$, obtained by applying a decelerative impulse at the apogee of $E_{0}$. For, the possible corner $S$ is in the domain where it is more economical to follow the line $S P$ to infinity rather than using the trajectory $S K$. In turn, the composite trajectory $E_{0} S P$ is less economical than the true optimal trajectory $E_{0} H$, since $E_{0}$ is on the other side of the separatrix.

3.4. Use of Atmospheric Drag. For a transfer between a point and a final set which constitutes orbits outside the atmospheric sphere of radius $R$ and when the change of orbital plane is not involved, we must always have $\beta \geqslant R$, as shown in Fig. 4 . The proof of the statement is very simple. Assume that the curve $A B C$ is a possible trajectory. Then, the Hohmann transfer $A B^{*} C$ is obviously superior. If the trajectory to be considered is the curve $D E G F$ with $G$ inside the atmosphere, then it is better to use $D E G^{*} F$, since the portion from $E$ to $F$ via $G^{*}$ can be realized without fuel consumption by using

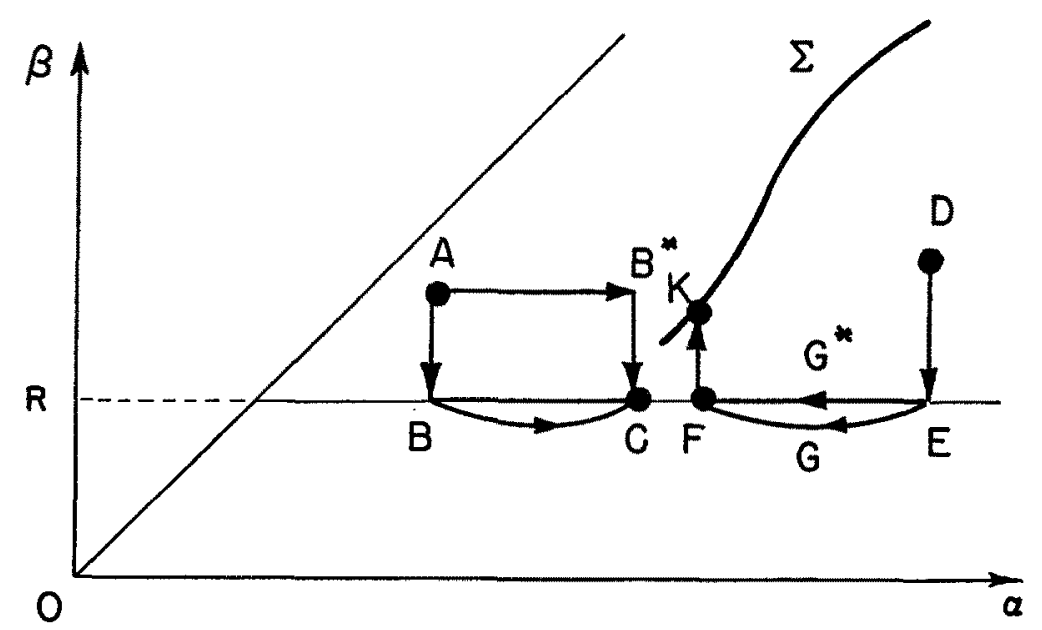

Fig. 4. Use of atmospheric braking. 
atmospheric braking at the perigee distance $\beta=R$ of the orbit $E$. We can notice that atmospheric braking is used only in the direction of decreasing $\alpha$.

If the final state $\Sigma$ does not intersect the line $\beta=R$, using atmospheric braking as part of the optimal process, the last corner $F$ can be found by minimizing the last impulse (to go from $F$ to $K$ )

$$
\Delta u=\sqrt{ }\left[2 \mu \beta_{1} / \alpha_{1}\left(\alpha_{1}+\beta_{1}\right)\right]-\sqrt{ }\left[2 \mu R / \alpha_{1}\left(\alpha_{1}+R\right)\right]
$$

subject to the constraint

$$
\theta\left(\alpha_{1}, \beta_{1}\right)=0
$$

If the final state intersects the line $\beta=R, F$ and $K$ coincide, and the last impulse is infinitesimal, just enough to bring the perigee distance of the final orbit above the level $R$, thus stopping the atmospheric braking. In this case, we can see that reaching the final state in the minimum time (here, minimum fuel consumption) is the same as optimally reaching the modified final state, which is composed of $\Sigma$ with $\beta_{1} \geqslant R$ and the line $\beta_{1}=R, \alpha_{1} \geqslant \alpha_{F}$.

3.5. Optimum Modes. The optimal trajectories in the $\alpha \beta$-plane (with $\alpha \geqslant \beta \geqslant R$ ) are always of one of the four following modes (Fig. 5):

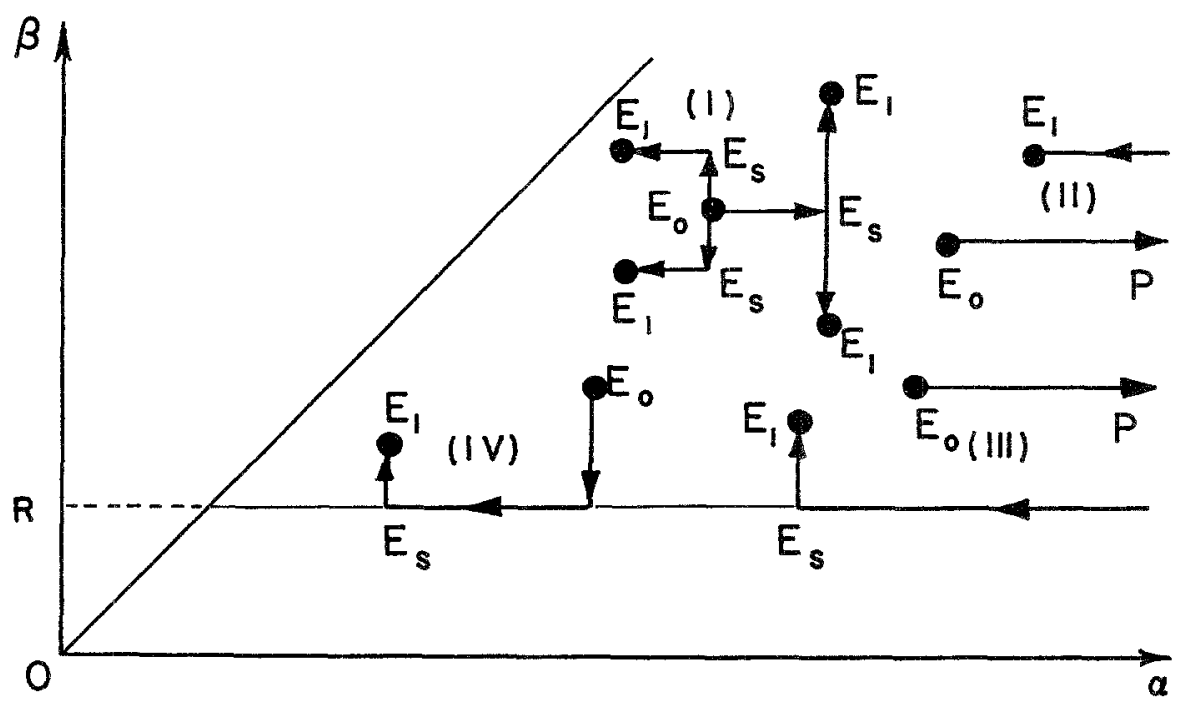

Fig. 5. Optimum modes. 
Case I: Hohmann Mode. Generally, this mode has two impulses and one intermediary orbit $E_{S}$ with

$$
\alpha_{S}=\max \left(\alpha_{0}, \alpha_{1}\right), \quad \beta_{S}=\left\{\begin{array}{lll}
\beta_{0} & \text { if } & \alpha_{S}=\alpha_{1} \\
\beta_{1} & \text { if } & \alpha_{S}=\alpha_{0}
\end{array}\right.
$$

$\left(\alpha_{0}, \beta_{0}\right)$ and $\left(\alpha_{1}, \beta_{1}\right)$ being the initial and final points. The switching at $\left(\alpha_{S}, \beta_{S}\right)$ is either of the $D D$-type $\left(\alpha_{1}<\alpha_{0}, \beta_{1}<\beta_{0}\right)$ or the $A A$-type $\left(\alpha_{1}>\alpha_{0}, \beta_{1}>\beta_{0}\right)$ or one of the two $A D$-types. The corner $E_{S}$ is either on the switching curve or is such that $E_{1}$ is at one end of the final state, when it is constrained.

We shall see that sometimes the Hohmann mode degenerates into a one-impulse-at-the-perigee mode $\left(\beta_{0}=\beta_{1}\right)$ or a one-impulse-at-the-apogee mode $\left(\alpha_{0}=\alpha_{1}\right)$ or a parabolic mode $\left(\alpha_{1}=+\infty\right)$.

Case II: Biparabolic Mode. There exists an infinitesimal impulse at infinity to transfer the vehicle from one parabola to the other. The total characteristic velocity for the transfer is

$$
u_{\mathrm{II}}=\sqrt{ }\left(2 \mu / \beta_{0}\right)-\sqrt{ }\left[2 \mu \alpha_{0} / \beta_{0}\left(\alpha_{0}+\beta_{0}\right)\right]+\sqrt{ }\left(2 \mu / \beta_{1}\right)-\sqrt{ }\left[2 \mu \alpha_{1} / \beta_{1}\left(\alpha_{1}+\beta_{1}\right)\right]
$$

Case III: Parabolic Mode with Atmospheric Braking. The apogee of the intermediary orbit is theoretically at infinity and the total characteristic velocity is

$$
\begin{aligned}
u_{\mathrm{III}}= & \sqrt{ }\left(2 \mu / \beta_{0}\right)-\sqrt{ }\left[2 \mu \alpha_{0} / \beta_{0}\left(\alpha_{0}+\beta_{0}\right)\right] \\
& +\sqrt{ }\left[2 \mu \beta_{1} / \alpha_{1}\left(\alpha_{1}+\beta_{1}\right)\right]-\sqrt{ }\left[2 \mu R / \alpha_{1}\left(\alpha_{1}+R\right)\right]
\end{aligned}
$$

The corner $E_{S}$ is found by minimizing the last impulse subject to the constraint $\theta\left(\alpha_{1}, \beta_{1}\right)=0$.

Case IV: Two-Impulse Mode with Atmospheric Braking. This mode of course requires $\alpha_{1}<\alpha_{0}$. The total characteristic velocity is

$$
\begin{aligned}
u_{\mathrm{TV}}= & \sqrt[V]{ }\left[2 \mu \beta_{0} / \alpha_{0}\left(\alpha_{0}+\beta_{0}\right)\right]-\sqrt{ }\left[2 \mu R / \alpha_{0}\left(\alpha_{0}+R\right)\right] \\
& +\sqrt{ }\left[2 \mu \beta_{1} / \alpha_{1}\left(\alpha_{1}+\beta_{1}\right)\right]-\sqrt{ }\left[2 \mu R / \alpha_{1}\left(\alpha_{1}+R\right)\right]
\end{aligned}
$$

The corner $E_{S}$ is found by minimizing the last impulse. This impulse is infinitesimal if the final state intersects the line $\beta=R$. 
If $\left(\alpha_{0}, \beta_{0}\right)$ and $\left(\alpha_{1}, \beta_{1}\right)$ are known, it is easy to compare the four optimal possibilities. For example, we may use the following conditions.

Mode I requires

$$
\max \left(\beta_{0}, \beta_{1}\right) \leqslant 11.938 \min \left(\beta_{0}, \beta_{1}\right)
$$

Mode II requires

$$
\max \left(\beta_{0}, \beta_{1}\right)>9 \min \left(\beta_{0}, \beta_{1}\right), \quad \beta_{1} \geqslant 4 R\left(\alpha_{1}+R\right) / \alpha_{1}
$$

Mode III requires

$$
\begin{aligned}
& \beta_{1} \leqslant 4 R\left(\alpha_{1}+R\right) / \alpha_{1} \Rightarrow \beta_{1} \leqslant(2+2 \sqrt{ } 2) R \\
& \beta_{0}>4 R\left\{1+\left[R / \max \left(\alpha_{0}, \alpha_{1}\right)\right]\right\}
\end{aligned}
$$

Mode IV requires

$$
\begin{gathered}
\alpha_{1}<\alpha_{0} \\
\beta_{0} \leqslant 4 R\left(\alpha_{0}+R\right) / \alpha_{0} \Rightarrow \beta_{0} \leqslant(2+2 \sqrt{ } 2) R \\
\alpha_{1}^{2}\left(4 R-\beta_{1}\right)+\alpha_{1} R\left(4 R+\beta_{1}\right)+R^{2}\left(R+\beta_{1}\right)>0 \Rightarrow \beta_{1}<5.879 R
\end{gathered}
$$

These conditions are always sufficient to compare at least Modes II, III, IV.

\section{Applications}

The foregoing analysis is applied in this section to solve several problems of orbit correction. The final state can be a portion of a curve, a curve with infinite branch, or a composite curve. It is denoted by the symbol $\Sigma$. In the first three examples, the existence of a corner on a switching curve is ruled out by using the separatrix as a curve of comparison. In the last two examples, a corner exists for certain types of transfer.

4.1. Change in the Apogee. Let $d$ be the final apogee distance. The final state is a segment of a straight line parallel to the $\beta$-axis (Fig. 6)

$$
\alpha_{1}=d \geqslant \beta_{1}>R
$$

If $\alpha_{0}<d$, the optimum mode is the one-impulse mode, accelerative at the perigee (orbit 1). If $\alpha_{0}>d$, there are four possible optimal trajectories:

(a) Hohmann type, bringing the vehicle to the circular orbit $C$ of radius $d$. This transfer occurs only if $\beta_{0}>d$ (orbit 2). 


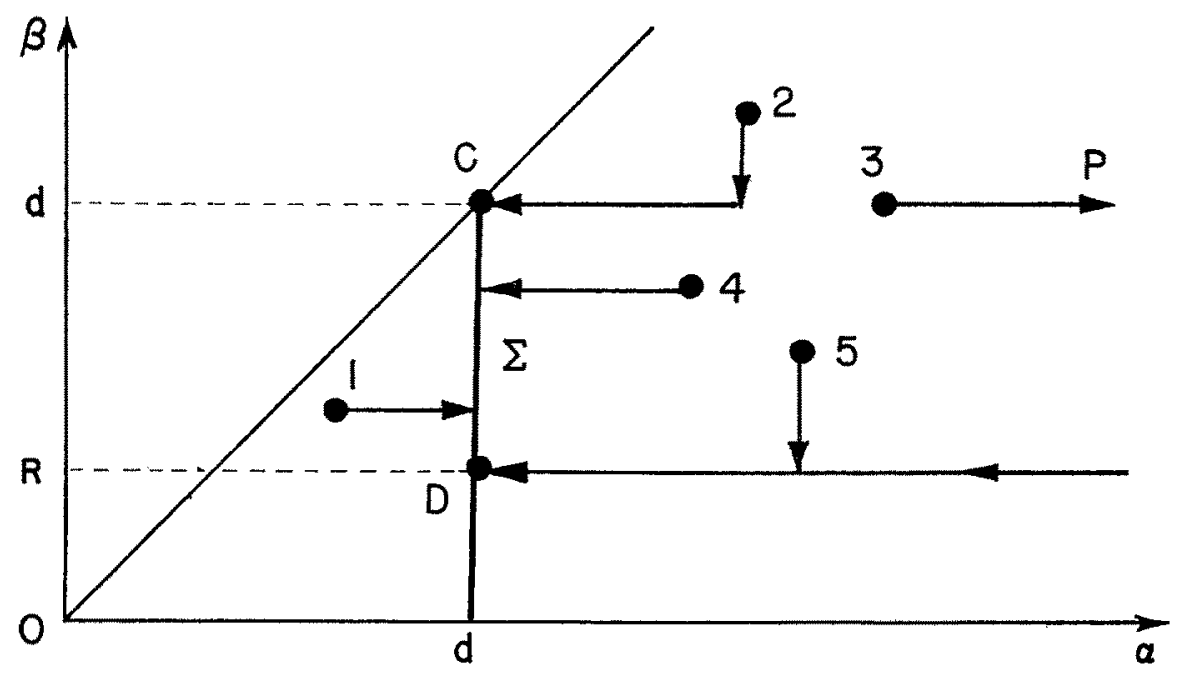

Fig. 6. Change in the apogee.

(b) Parabolic mode with atmospheric braking (trajectory from 3 to $D$ ). This mode occurs only when

$$
\beta_{0}>4 R\left(\alpha_{0}+R\right) / \alpha_{0}
$$

(c) One-impulse mode, decelerative at the perigee (orbit 4). This mode occurs only when

$$
\alpha_{0}>d \geqslant \beta_{0}
$$

(d) Two-impulse mode with atmospheric braking (trajectory from 5 to $D$ ).

4.2. Change in the Perigee. Let $d$ be the final perigee distance. The final state is a ray parallel to the $\alpha$-axis (Fig. 7)

$$
\alpha_{1} \geqslant \beta_{1}=d>R
$$

If $\beta_{0}>d$, there are two possible trajectories:

(a) If $\beta_{0} \leqslant 4 d\left(\alpha_{0}+d\right) / \alpha_{0}$, the optimum mode is the one-impulse mode, decelerative at the apogee (orbit 1 ).

(b) If $\beta_{0} \geqslant 4 d\left(\alpha_{0}+d\right) / \alpha_{0}$, the optimum mode is parabolic (orbit 2). 


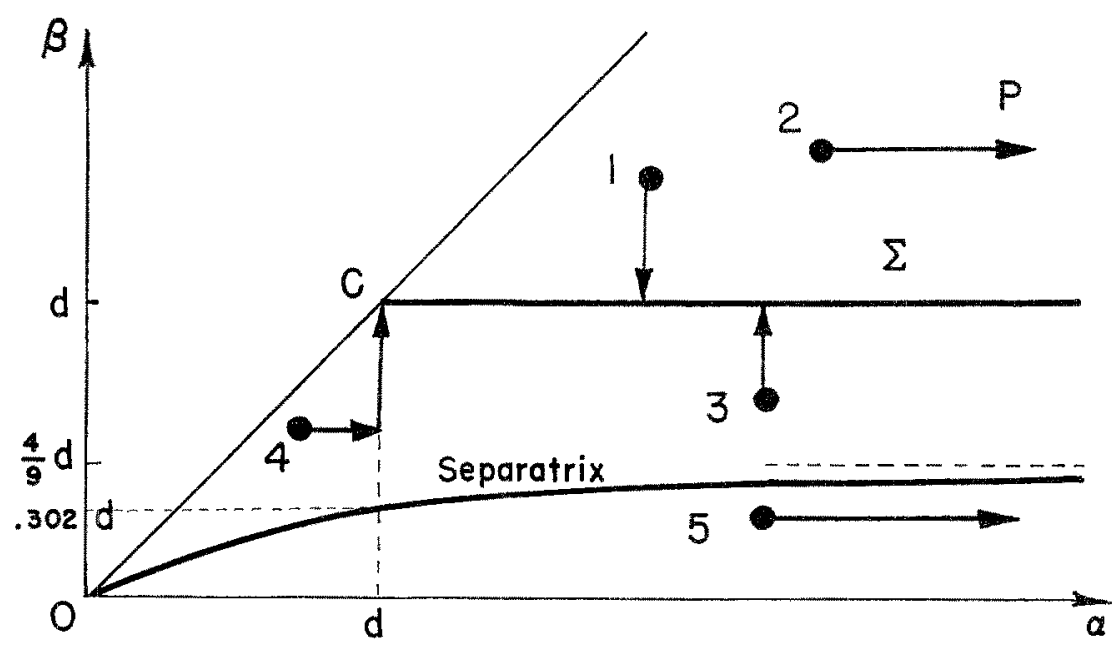

Fig. 7. Change in the perigee.

If $\beta_{0}<d$, there are three possible trajectories:

(a) One-impulse mode, accelerative at the apogee (orbit 3). This mode is optimum when

$$
\begin{gathered}
\alpha_{0} \geqslant d \\
\alpha_{0} \beta_{0}\left(3 \alpha_{0}-\beta_{0}\right)^{2}-4 \alpha_{0} d\left(\alpha_{0}-\beta_{0}\right)\left(\alpha_{0}-3 \beta_{0}\right)-4 d^{2}\left(\alpha_{0}-\beta_{0}\right)^{2} \geqslant 0
\end{gathered}
$$

If $\beta_{0} \geqslant 4 d / 9$, the second inequality is automatically satisfied.

(b) Hohmann transfer (from 4 to $C$ ). This mode is optimum when

$$
0.3026 d \leqslant \beta_{0} \leqslant \alpha_{0}<d
$$

(c) Parabolic mode (orbit 5).

4.3. Change in the Eccentricity, Let $e_{1}$ be the final eccentricity. The final state is a straight line (Fig. 8), that is,

$$
\beta_{1}=k_{1} \alpha_{1}, \quad k_{1}=\left(1-e_{1}\right) /\left(1+e_{1}\right)
$$

If $e_{0}<e_{1}$, the optimum mode is the one-impulse mode, accelerative at the perigee (orbit 1). If $e_{0}>e_{1}$, there are four possible trajectories:

(a) One-impulse mode, accelerative at the apogee (orbit 2). This mode occurs only when

$$
k_{1} \leqslant\left[\left(1+k_{0}\right) / 2\left(1-k_{0}\right)\right]\left[1+\sqrt[V]{ }\left(1+k_{0}\right)\right]-1
$$




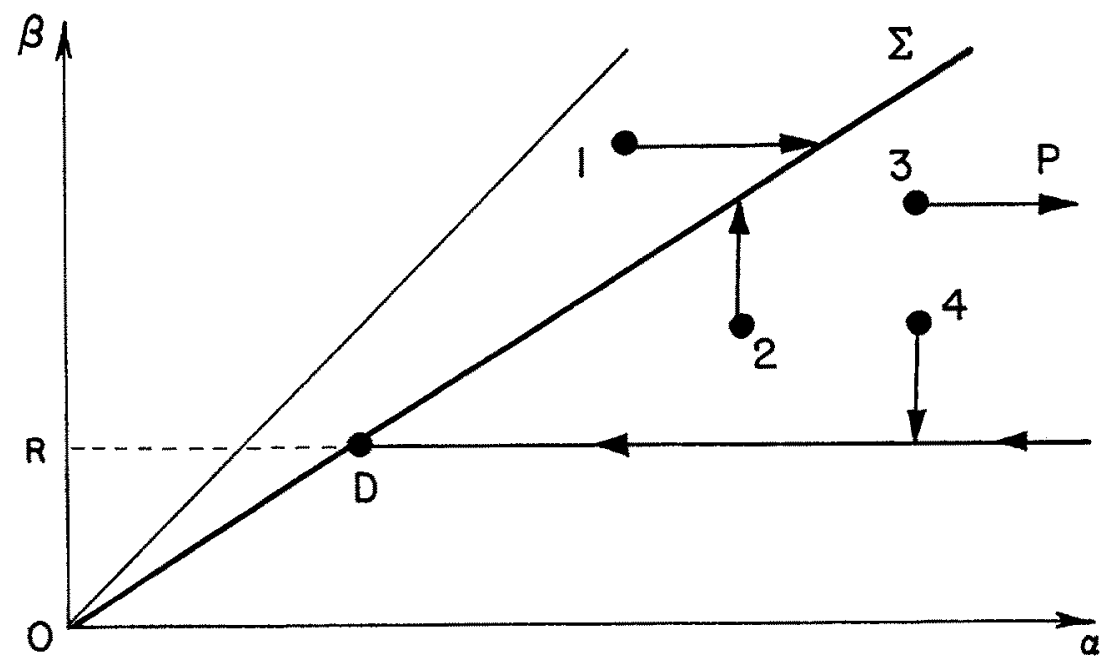

Fig. 8. Change in the eccentricity.

where

$$
k_{0}=\beta_{0} / \alpha_{0}=\left(1-e_{0}\right) /\left(1+e_{0}\right)
$$

(b) If the sign of Ineq. (47) is reversed (this requires $k_{0}<0.3026$, $e_{0}>0.53533$ ), the optimum mode can be the parabolic mode without atmospheric braking (since $\Sigma$ has infinite branch) or parabolic mode with atmospheric braking (trajectory from 3 to $D$ ).

(c) Two-impulse mode with atmospheric braking (orbit 4 to $D$ ). The second impulse is infinitesimal. This mode occurs only when

$$
\beta_{0} \leqslant 4 R\left(\alpha_{0}+R\right) / \alpha_{0}
$$

4.4. Change in the Major Axis. Let $d$ be the final major axis. The final state is a segment of a straight line (Fig. 9)

$$
\alpha_{1}+\beta_{1}=d, \quad \alpha_{1} \geqslant \beta_{1}>R
$$

If $\alpha_{0}+\beta_{0}<d$, the optimum mode is the one-impulse mode, accelerative at the perigee (orbit 1). If $\alpha_{0}+\beta_{0}>d$, there are four possible trajectories:

(a) Two-impulse mode with switching of the $D D$-type (orbit 2). The perigee distance of the intermediary orbit is

$$
\beta_{S}=\left\{-3 \alpha_{0}\left(\alpha_{0}-d\right)+2 \alpha_{0} \sqrt{ }\left[d\left(2 \alpha_{0}+3 d\right)\right]\right\} /\left(9 \alpha_{0}+d\right)
$$




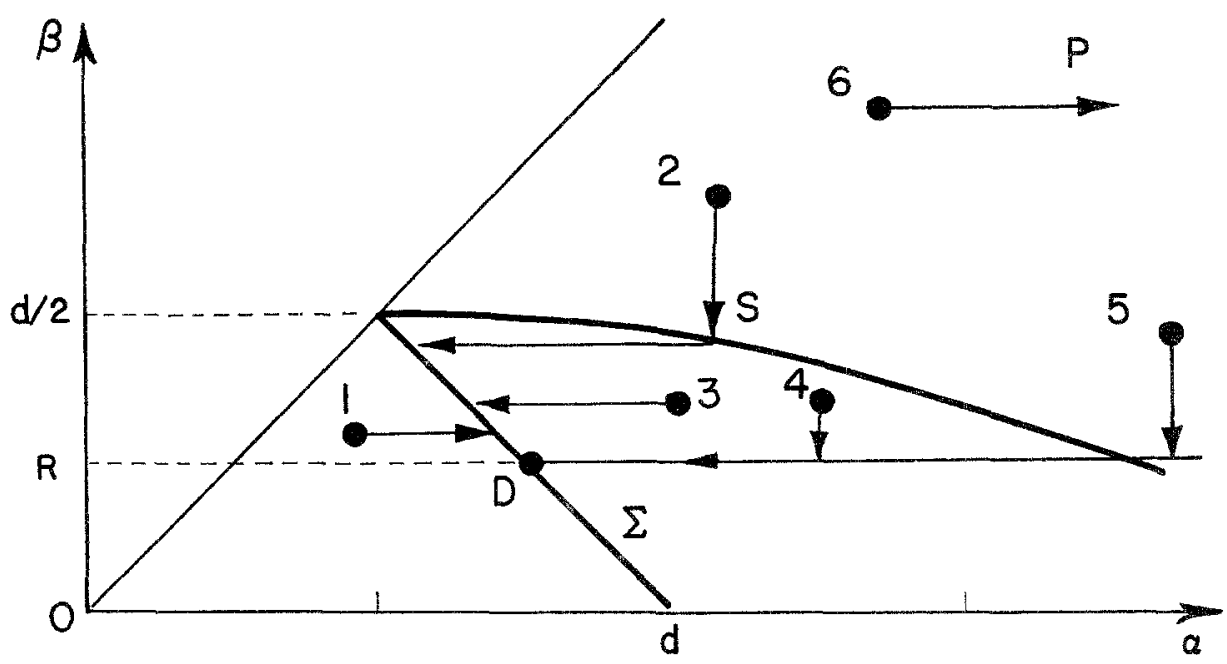

Fig. 9. Change in the major axis.

This mode occurs only when

$$
\beta_{0}>\beta_{S}>R
$$

The equality $\beta_{0}=\beta_{S}$, written without the subscript 0 , is the equation of the switching curve.

(b) One-impulse mode, decelerative at the perigee (orbit 3). This mode occurs only when

$$
R<\beta_{0} \leqslant \beta_{S}
$$

(c) Two-impulse mode with atmospheric braking (orbit 4 and 5 to $D$ ). The second impulse is infinitesimal.

(d) Parabolic mode with atmospheric braking (orbit 6 to $D$ ). This mode occurs only when

$$
\beta_{0}>4 R\left(\alpha_{0}+R\right) / \alpha_{0}
$$

4.5. Change in the Angular Momentum. This is the same as changing the semilatus rectum. Let $2 d$ be the final value of the semilatus rectum. The final state is a branch of hyperbola (Fig. 10)

$$
\beta_{1}=\alpha_{1} d\left(\alpha_{1}-d\right), \quad \alpha_{1} \geqslant 2 d \geqslant \beta_{1}>d
$$




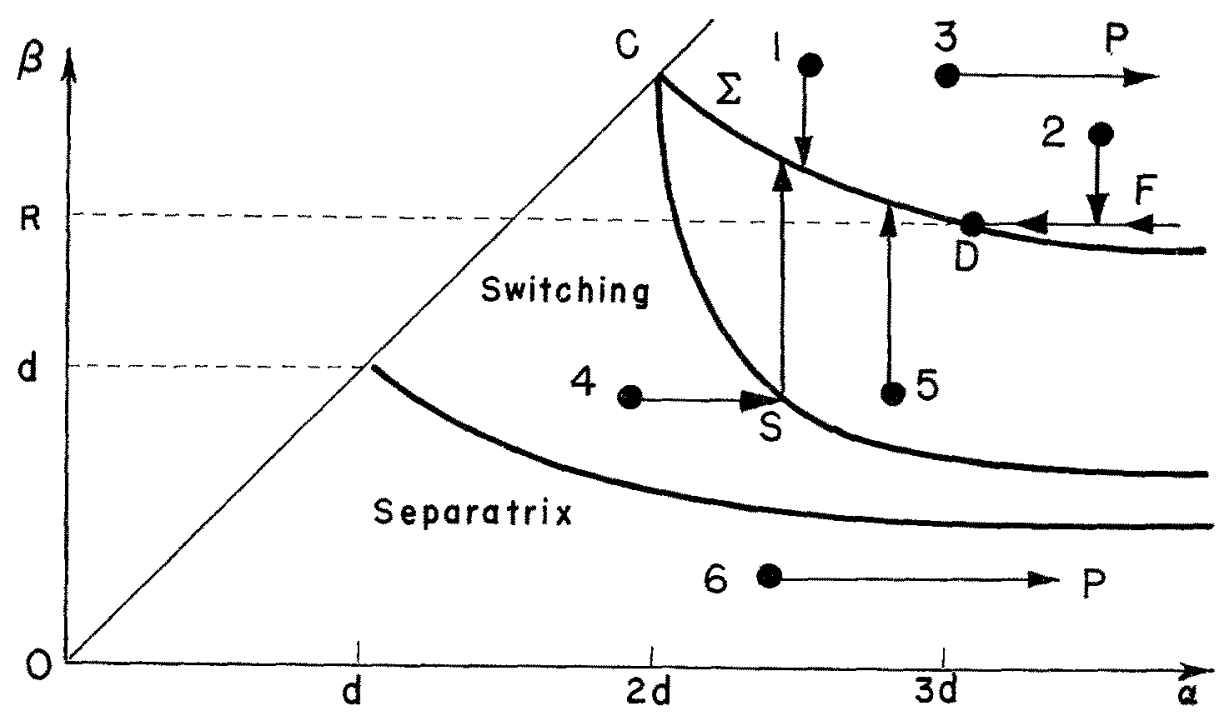

Fig. 10. Change in the angular momentum.

If the initial angular momentum is larger than the final angular momentum, that is, if

$$
\beta_{0}>\alpha_{0} d /\left(\alpha_{0}-d\right)
$$

there are four possible modes:

(a) One-impulse mode, decelerative at the apogee (orbit 1).

(b) Two-impulse mode with atmospheric braking (orbit 2 to $D$ ). This mode occurs and is optimum when

$$
d<R<2 d, \quad \alpha_{0} \geqslant \alpha_{D}=R d /(R-d), \quad \beta_{0} \leqslant 4 R\left(\alpha_{0}+R\right) / \alpha_{0}
$$

(c) Parabolic mode with atmospheric braking (orbit 3 to $D$ ). This mode occurs only when

$$
d<R<2 d, \quad \beta_{0} \geqslant 4 R\left(\alpha_{0}+R\right) / \alpha_{0}
$$

(d) Parabolic mode without atmospheric braking. This mode occurs and is optimum when

$$
R<d, \quad \beta_{0} \geqslant 4 \alpha_{0}^{2} d /\left(\alpha_{0}-d\right)^{2}
$$

We notice that, in the case where Ineq. (56) and the first of Ineqs. (57) are verified, the strategy is to go in an optimum way from the initial orbit to the final state represented by the composite curve $C D F$. 
If the sign of Ineq. (56) is reversed, that is, if the initial angular momentum is less than the final angular momentum, there are three possible modes, none of them involving atmospheric braking:

(a) Two-impulse mode with switching (orbit 4). This mode occurs and is optimum when

$$
\begin{gathered}
\beta_{0}>(4 / 9) d, \\
S\left(\alpha_{0}, \beta_{0}\right)=\left(9 \beta_{0}-4 d\right) \alpha_{0}{ }^{3}+6 \beta_{0}\left(\beta_{0}-2 d\right) \alpha_{0}{ }^{2}+\beta_{0}{ }^{2}\left(\beta_{0}-12 d\right) \alpha_{0}-4 \beta_{0}{ }^{3} d \leqslant 0
\end{gathered}
$$

The equality written without the subscript 0 is the equation of the switching curve. The apogee distance of the intermediary orbit is obtained by solving $S\left(\alpha_{S}, \beta_{0}\right)=0$.

(b) When $S\left(\alpha_{0}, \beta_{0}\right) \geqslant 0$, the optimum mode is the one-impulse mode, accelerative at the apogee (orbit 5 ).

(c) Parabolic mode (orbit 6). This mode occurs and is optimum when

$$
R<\beta_{0} \leqslant(4 / 9) d
$$

\section{Conclusion}

This paper presents the complete analytical solution of several fundamental problems in orbital correction. The initial state is a given point in the phase space, while the terminal state is a segment of a curve, a branch of a curve, or a composite curve. The possible use of atmospheric braking is discussed; and, by modifying the final state to include the line $\beta=R$, the problem again can be solved by the same method. The selection of the apogee and perigee distances as state variables gives a symmetric form to the problem and results in a linear differential equation of the first order for the ratio of the adjoint variables.

The applications of the solution derived in this paper are not restricted to the examples which have been selected. The solution can be applied to the problem of optimum disorbit (Ref. 3), optimum ascent into an orbit (Ref. 4), or optimum orbit correction involving more than two orbital elements (Ref. 5).

\section{References}

1. Moulton, F. R., An Introduction to Celestial Mechanics, The Macmillan Company, New York, 1914. 
2. Leitmann, G., An Introduction to Optimal Control, McGraw-Hill Book Company, New York, 1966.

3. Busemann, A., and VInH, N. X., Optimum Disorbit by Multiple Impulses, Journal of Optimization Theory and Applications, Vol. 2, No. 1, 1968.

4. Marchal, C., Optimization de la Phase Extra-Atmosphérique de la Montée en Orbite, Première Partie, La Recherche Aerospatiale, Vol. 1, No. 116, 1967.

5. Edelbaum, T. N., Propulsion Requirements for Controllable Satellites, ARS Journal, Vol. 31, No. 8, 1961. 